\title{
Performance of the CMS Silicon Tracker
}

\section{Matthew Chan ${ }^{* \dagger}$}

Massachusetts Institute of Technology

E-mail: Matthew.Chan@cern.ch

The CMS tracker is the largest silicon detector ever built, covering 200 square meters and providing an average of 14 high-precision measurements per track. Data from proton-proton collisions at a center-of-mass energy of $7 \mathrm{TeV}$ are used to measure the performance of the detector and the reconstruction algorithms. The resolution and efficiency of the track, vertex, and beam line reconstruction are measured in data and compared to the results from simulation. An example of the culmination of this effort is shown in the performance of the b-tagging algorithms, critical to many physics analyses at CMS.

10th International Conference on Large Scale Applications and Radiation Hardness of Semiconductor Detectors

6-8 July 2011

Florence, Italy

\footnotetext{
*Speaker.

${ }^{\dagger}$ On behalf of the CMS Collaboration
} 


\section{Introduction}

The Compact Muon Solenoid (CMS) is a general purpose detector at the Large Hadron Collider at CERN. The CMS magnet generates a $3.8 \mathrm{~T}$ solenoidal magnetic field that bends the trajectories of charged particles produced in the proton-proton collisions at the center of the detector. The momenta of charged particles can be inferred from the curvature of their paths as they travel through the CMS Tracker. The Tracker is the innermost sub-detector of CMS and comprises a 3layer pixel detector and a 10-layer silicon strip detector [1]. Both the pixel and strip detectors have end cap disks to provide tracking in the forward and backward regions of CMS. Tracking coverage extends out to $|\eta|=2.5$. The pixel detector has an inner radius of $4.4 \mathrm{~cm}$ and an outer radius of $10.2 \mathrm{~cm}$, while the strip tracker has an inner radius of $25 \mathrm{~cm}$ and an outer radius of $1.1 \mathrm{~m}$. Track reconstruction is seeded by pixel hits and then extrapolated out to compatible hits in the silicon strip tracker. Strip hits are added according to a pattern recognition algorithm based on the Kalman filter [2]. After the track hits are identified, a fit is performed to obtain the overall track parameters. The tracking algorithm then reiterates on the remaining hits until all hits have been associated with a track or determined to be spurious.

Reconstructed tracks are used to identify leptons, charged hadrons, and jets. Tracks are also used to reconstruct the primary vertex of each proton-proton collision. As these are essential components of nearly all CMS physics analyses, it is important to reconstruct tracks with high efficiency and momentum resolution. The efficiency and resolution of track and primary vertex reconstruction have been measured using data-driven methods. Tracks can also be used to identify b quark jets; the performance of these b-tagging algorithms is reviewed.

\section{Tracking Efficiency}

Tracking efficiency is the probability that a charged particle passing through the Tracker is successfully reconstructed. It is a key measure of the performance of the Tracker. The tracking efficiency for pions and muons was assessed by embedding simulated tracks in data events and testing if the tracks could be reconstructed. Furthermore, tracking efficiency of muons was measured in $\mathrm{J} / \psi \rightarrow \mu \mu$ events using a method called tag-and-probe [3].

\subsection{Embedded Tracks}

Tracking efficiency was measured by simulating the traversal of pions and muons through the Tracker using GEANT4 and measuring the probability of reconstructing tracks from the resulting hits. To incorporate realistic noise and background effects, these simulated tracks were embedded in data events taken with a minimum-bias trigger [3]. This embedding method has the advantage of allowing acceptance and reconstruction effects to be studied separately. The probability that a simulated track will produce enough hits in the tracker to be reconstructed can be measured before and after incorporating the effect of hit occupancy from low-momentum particles present in minimum-bias data. Figure 1 shows the tracking efficiency for pions and muons, both for simulated tracks embedded in data events and for completely simulated events. The tracking efficiency is over $99 \%$ for both muons and pions in the studied momentum range. 


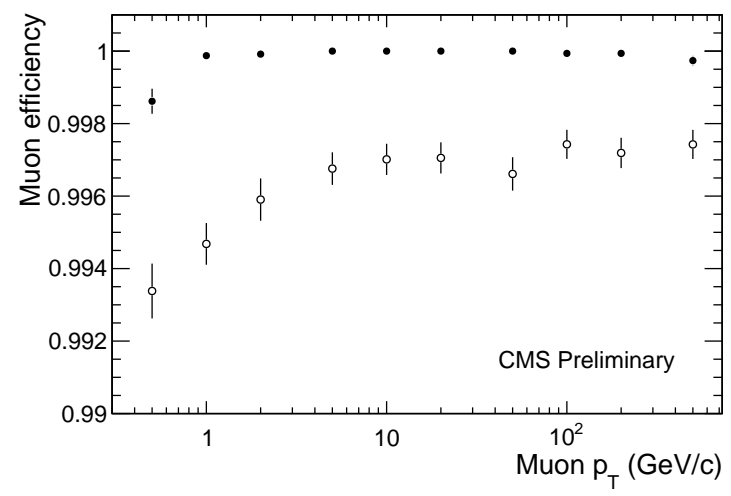

(a)

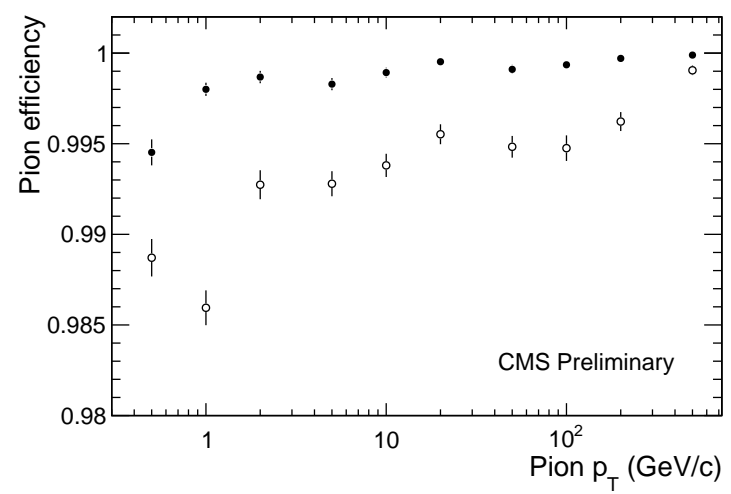

(b)

Figure 1: Tracking efficiency measured by embedding simulated tracks in data events (open circles) for muons (a) and pions (b). Tracking efficiency measured in simulated events (solid circles) are also shown for comparison.

\subsection{Muon Tag and Probe}

Tracking efficiency for muons was further studied by the tag-and-probe method. This method exploits the presence of the two real muons in a $\mathrm{J} / \psi \rightarrow \mu \mu$ event. $\mathrm{J} / \psi$ events are selected by identifying one high quality muon (the tag) and one looser quality muon (the probe). The tag muon must be reconstructed in both the Tracker and the muon system and pass stringent quality requirements on the number of hits and the goodness of the track fit, while the probe muon need only be reconstructed in the muon system. The purpose of the tag muon is to increase confidence that the selected event contains a $\mathrm{J} / \psi$ decay. The probe muon is used to measure the tracking efficiency by testing whether it was successfully reconstructed in the Tracker. Once the tag-and-probe dimuon spectra are created, the number of $J / \psi$ events is extracted by fitting the mass distribution with a Gaussian plus a third-order Chebyshev polynomial, where the Gaussian models the $J / \psi$ line shape and the Chebyshev polynomial models the background. The normalization of the fitted Gaussian gives the number of $\mathrm{J} / \psi$ events among the selected dimuon events. The tracking efficiency (relative to the efficiency of the muon chambers) is given by the number of probe muons reconstructed in the Tracker divided by the total number of probe muons.

The tag-and-probe method was applied to $125 \mathrm{nb}^{-1}$ of data. Muons were required to have $p_{T}>1.5 \mathrm{GeV} / \mathrm{c}$. Figure 2 shows the muon efficiency as a function of muon $\eta$ and as a function of the number of reconstructed primary vertices in the event. Muon tracking efficiency, given that the muon produced hits in the muon system, was found to be above $98 \%$ in all regions of the Tracker.

\section{Momentum Resolution}

Once charged particles have been identified, the Tracker needs to precisely measure the momentum of those particles based on their trajectories in the $3.8 \mathrm{~T}$ magnetic field generated by the CMS magnet. The momentum resolution is an important indicator of the performance of the CMS Tracker. The momentum resolution can be extracted from data by analyzing the width of the $J / \psi$ 


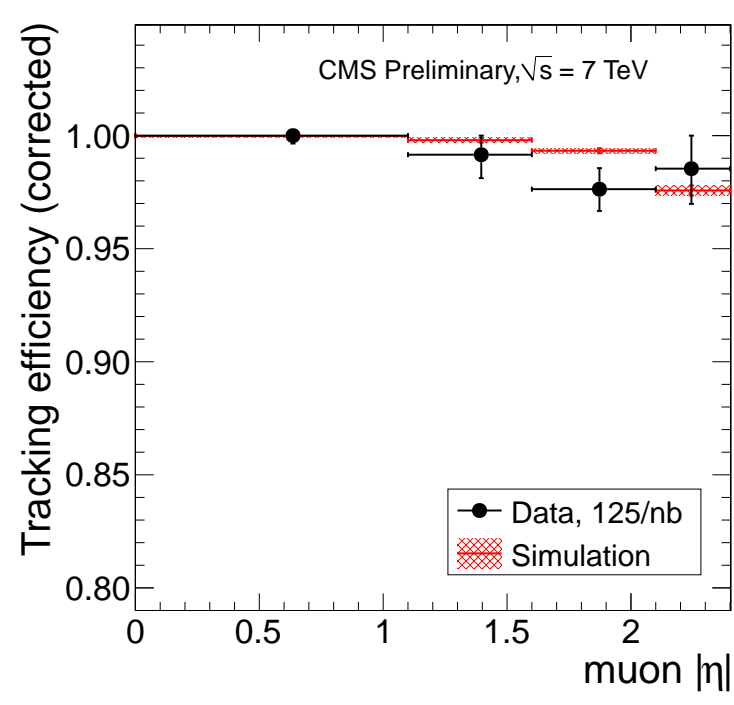

(a)

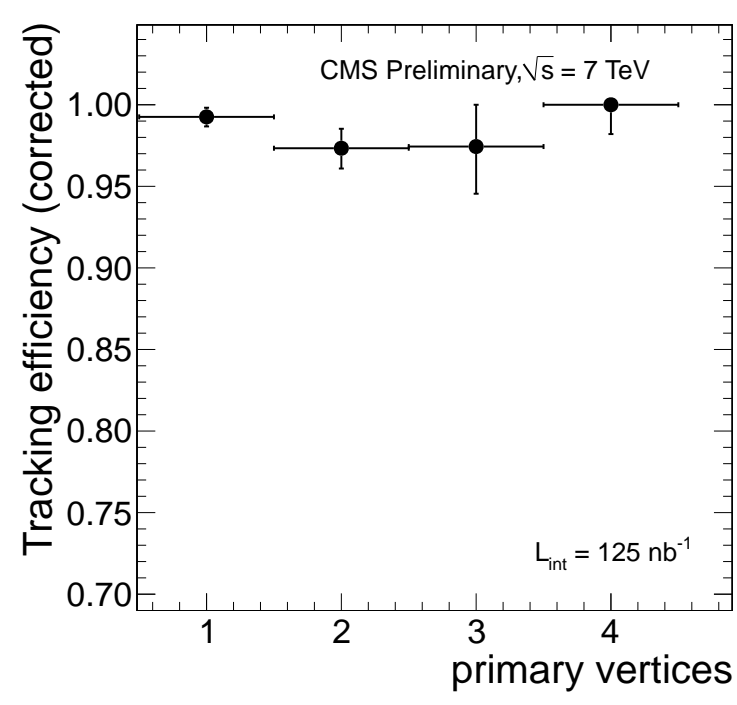

(b)

Figure 2: Muon efficiency measured by the tag and probe method as a function of $\eta$ (a) and of the number of primary vertices (b).

mass lineshape, where the $\mathrm{J} / \psi$ decays are reconstructed from muon pairs [4]. A higher momentum resolution results in a narrower mass peak, while a lower resolution is indicated by a broader mass peak. A shift of the mass peak from the nominal $\mathrm{J} / \psi$ mass indicates a momentum scale error. The mass peak is modeled by a Crystal Ball convoluted with a Gaussian, plus an exponential background. A likelihood fit in which the momentum scale and resolution of individual tracks are allowed to float is performed on the $\mathrm{J} / \psi$ lineshape as a function of track kinematics. Figure 3 shows the momentum resolution obtained from the fit on $40 \mathrm{nb}^{-1}$ of data. The resolution is found to between $1-2 \%$ for the central region and rises to $3 \%$ at high $\eta$.

\section{Primary Vertex Resolution}

The tracks observed by the Tracker are crucial to the reconstruction of the $p p$ collision vertex. The position of the primary vertex is found by applying an adaptive vertex fit on clusters of tracks within $z_{\text {sep }}=1 \mathrm{~cm}$ of each other. The resolution of the position determination is measured from data using the split method. In this method, the tracks associated to the vertex are randomly split into two equal groups. Each group of tracks is independently fit with the adaptive vertex fitter. The primary vertex position resolution can then be inferred from the difference between the two vertex fits. The vertex position resolution was determined for a range of track multiplicities. Figure 4 shows the primary vertex position resolution for the transverse and longitudinal axes. For primary vertices with more than 30 tracks, the position resolution is approximately $25 \mu \mathrm{m}$.

\section{B-Tagging}

Apart from its primary task of measuring the momentum of charged particles, the Tracker 


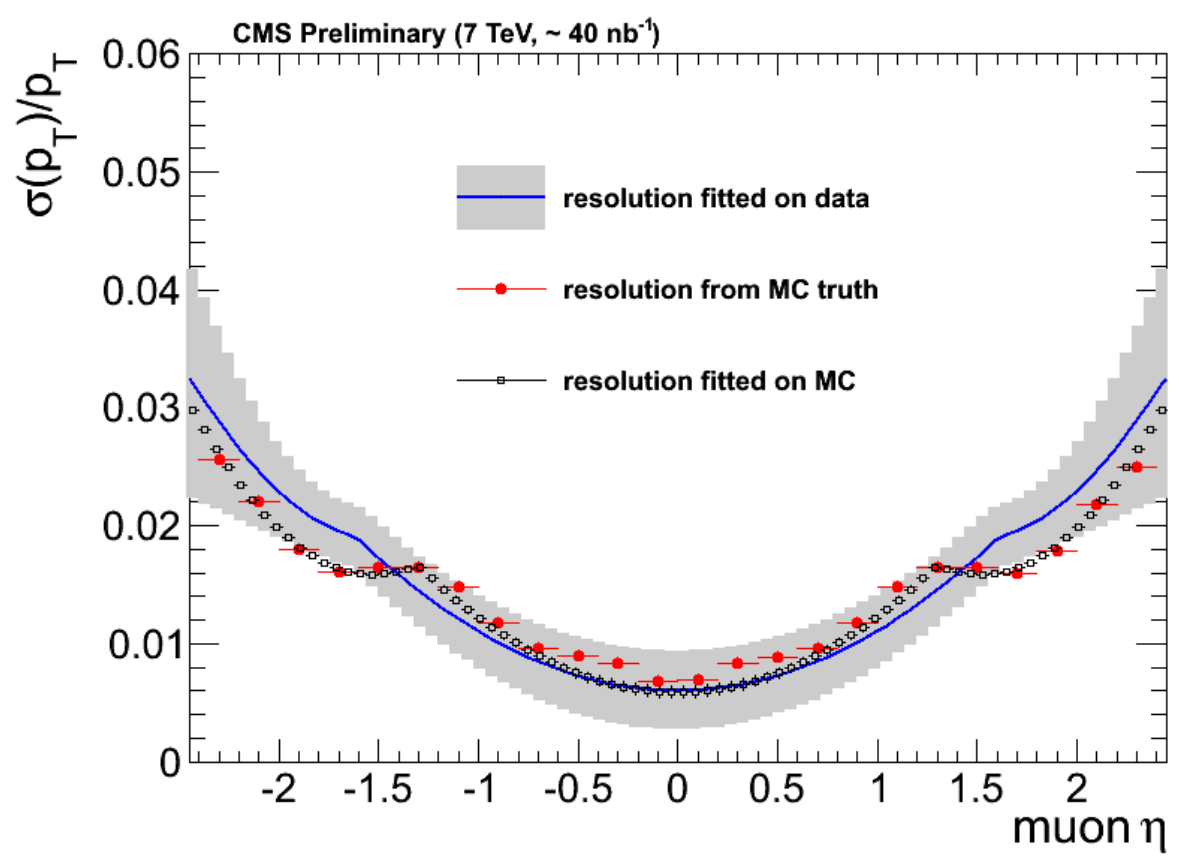

Figure 3: Momentum resolution obtained from a fit on the $\mathrm{J} / \psi$ mass peak. Results for the fit on data and Monte Carlo are both shown, as well as the exact resolution computed from Monte Carlo generator-level information.

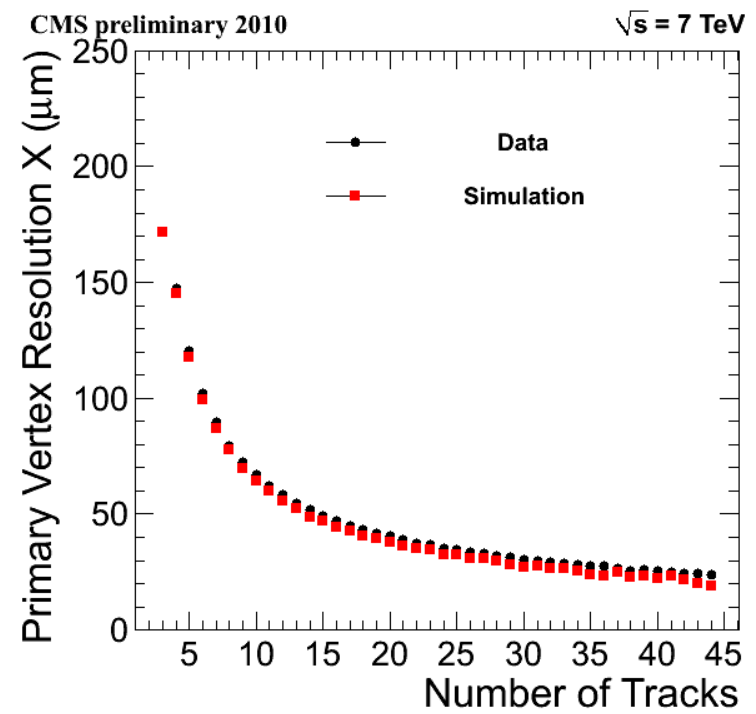

(a)

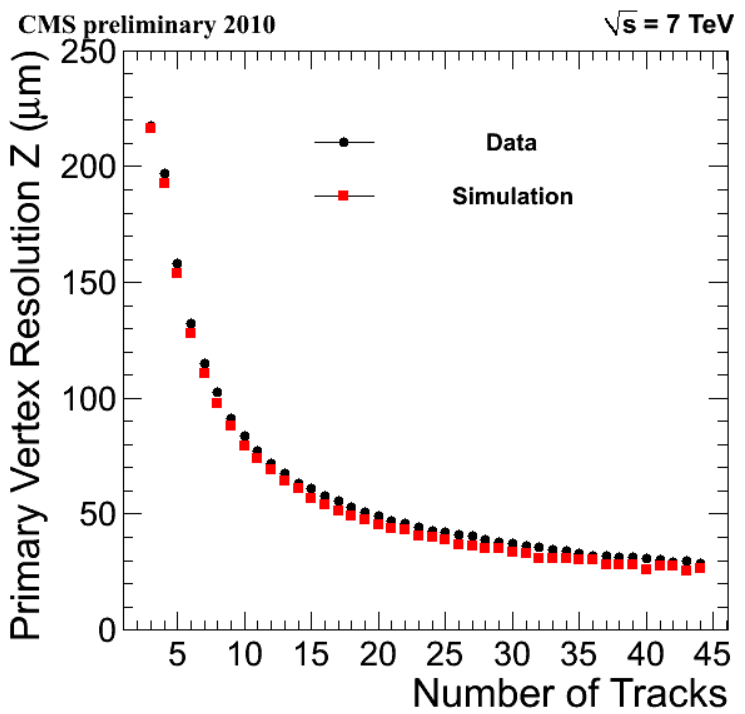

(b)

Figure 4: Primary vertex position resolution measured by the split method for the x-axis (a) and z-axis (b). 


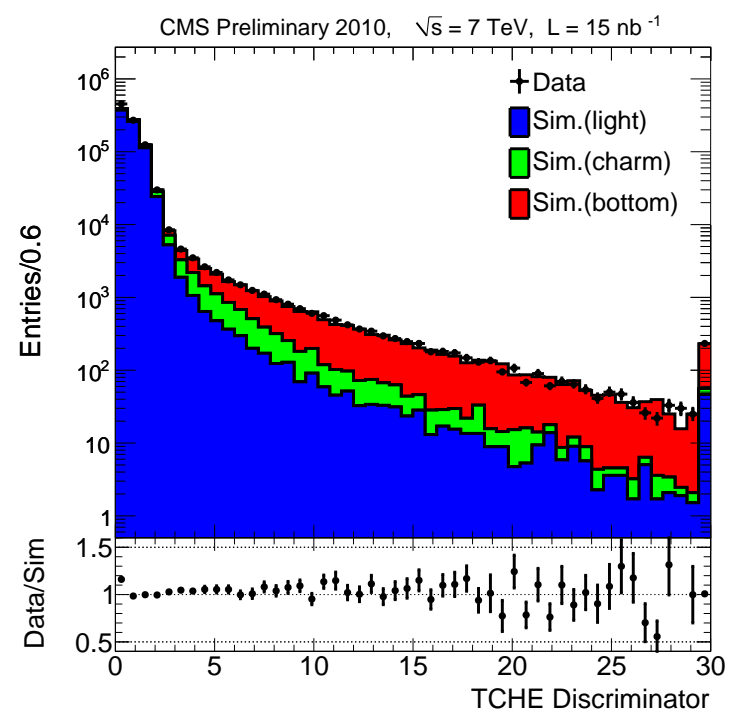

(a)

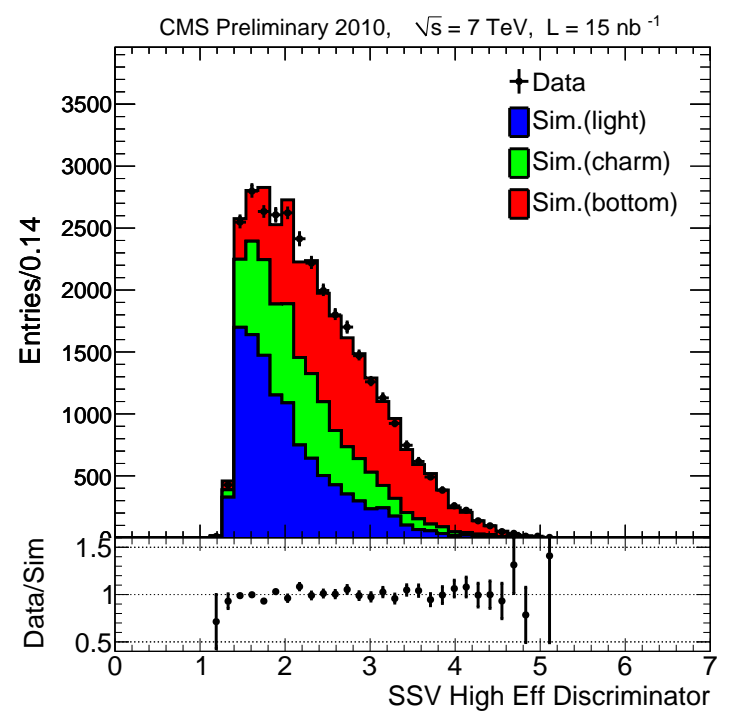

(b)

Figure 5: B-tagging discrimination variables. (a) The tracking counting b-tagging variable is defined as the third highest impact parameter significance among tracks in the jet. (b) The simple secondary vertex b-tagging variable is built from the 3D flight distance of the jet vertex.

is also essential to the identification of $\mathrm{b}$ quark jets. The process of determining whether a jet proceeded from a $\mathrm{b}$ quark is known as b-tagging. The performance of CMS's b-tagging algorithms exemplifies the Tracker's superb track resolution. B-tagging relies on the relatively long life time of $B$ mesons compared to hadrons formed from lighter quarks. Because of their long life times, $B$ mesons can travel an appreciable distance from the primary vertex before decaying into a jet. It is the displacement of the jet relative to the primary $p p$ vertex that experimentally distinguishes b-jets from ordinary gluon or light quark jets. The Tracker is sufficiently precise to resolve the distance between the displaced jet vertex and the primary vertex. The most relevant quantity used by b-tagging is the track impact parameter, which is defined as the distance of closest approach of the track to the primary vertex. Once the primary vertex is reconstructed from the prompt tracks of the collision as described in Section 4, the impact parameter of each track in a jet can be calculated. The larger the impact parameter, the more likely it is that the track originated from a secondary vertex, that is, that the jet is the product of a B meson. A variety of algorithms are currently under consideration for b-tagging. Two examples are the track counting and simple secondary vertex methods [6]. The track counting b-tagging method uses the number of tracks which exceed a certain impact parameter threshold. The greater the number of high impact parameter tracks, the greater the probability that the jet is a b-jet. The simple secondary vertex b-tagging method relies on the secondary vertex flight distance of the jet, where a large flight distance indicates a b-jet. Figure 5 shows the discrimination variables produced by the track counting and secondary vertex methods in $15 \mathrm{nb}^{-1}$ of data. They display significant distinguishing power in separating $b$-jets from light quark jets. Both of these methods depend on the ability of the Tracker to reconstruct track trajectories accurately enough to resolve the B meson decay length. 


\section{Conclusion}

The performance of the CMS Tracker was studied both in simulation and early collision data. Many of the Tracker's performance parameters were measured using data-driven methods that have minimal reliance on simulation, thereby sidestepping inaccuracies in simulation models. Nevertheless, results from simulation were found to be in good agreement with results from data. Tracking was shown to be highly efficient and precise for muons and pions, with efficiency above $99 \%$ and momentum resolution below 3\% for the kinematic range of interest to CMS physics analyses. The Tracker is also capable of accurately reconstructing primary vertices and resolving the displaced vertices of b-jets. The excellent performance of the Tracker will enable CMS to take full advantage of the ever increasing luminosity of the LHC.

\section{References}

[1] CMS Collaboration, "The CMS Experiment at the CERN LHC”, JINST 3 (2008) S08004.

[2] CMS Collaboration, "Tracking and Vertexing Results from First Collisions", CMS Physics Analysis Summary CMS-PAS-TRK-10-001 (2010).

[3] CMS Collaboration, "Measurement of Tracking Efficiency", CMS Physics Analysis Summary CMS-PAS-TRK-10-002 (2010).

[4] CMS Collaboration, "Measurement of Momentum Scale and Resolution Using Low-Mass Resonances and Cosmic Ray Muons", CMS Physics Analysis Summary CMS-PAS-TRK-10-004 (2010).

[5] CMS Collaboration, "Tracking and Primary Vertex Results in First 7 TeV Collisions", CMS Physics Analysis Summary CMS-PAS-TRK-10-005 (2010).

[6] CMS Collaboration, "Commissioning of $b$-jet Identification with $p p$ Collisions at $\sqrt{s}=7 \mathrm{TeV} ", C M S$ Physics Analysis Summary CMS-PAS-BTV-10-001 (2010). 